\title{
Autocriticismo en Pacientes con Depresión y su Repercusión en el Abandono de Psicoterapias Breves: Explorando el Rol Mediador de la Alianza Terapéutica y el Rol Moderador de la Edad de los Pacientes
}

\section{Self-Criticism in Patients with Depression and its Impact on Dropout in Short-Term Psychotherapies: Exploring the Mediator Role of the Therapeutic Alliance and the Moderator Role of Patients' Age}

\author{
Augusto Mellado \\ Pontificia Universidad Católica de Chile \\ Nicolás Suárez \\ Pontificia Universidad Católica de Chile \\ Sergio Gloger \\ Universidad de Chile
}

\author{
Carola Pérez \\ Universidad del Desarrollo \\ Paula Dagnino \\ Universidad Alberto Hurtado \\ Mariane Krause \\ Pontificia Universidad Católica de Chile
}

\begin{abstract}
Considerando la perspectiva de las polaridades de la personalidad (Blatt, 2004, 2007, 2008), el objetivo de este estudio fue describir la relación entre los niveles de autocriticismo y dependencia (DEQ) en pacientes con depresión (BDI-I) y su abandono de la psicoterapia, explorando el rol mediador de la alianza terapéutica (WAI-P) y el rol moderador de la edad de los pacientes. Mediante un diseño longitudinal y un muestreo por conveniencia, se reclutaron 81 pacientes adultos con depresión que estaban en psicoterapia breve en un centro de salud privado de Santiago, Chile, de los cuales 22 abandonaron el tratamiento. Por medio de un modelo de regresión con interacción, los resultados indican que, controlando sexo, escolaridad y edad de los pacientes y años de experiencia de los terapeutas, a mayor autocriticismo mayor probabilidad de abandonar la psicoterapia, relación que no es explicada por la alianza terapéutica. La asociación entre autocriticismo y abandono no se observa en los pacientes más jóvenes (30 años), pero sí en aquellos de mayor edad (> 40 años). Además, a mayor dependencia menor probabilidad de abandonar la psicoterapia.
\end{abstract}

Palabras clave: depresión, autocriticismo, dependencia, alianza terapéutica, abandono de la psicoterapia

Considering the perspective of personality polarities (Blatt, 2004, 2007, 2008), the aim of this study was to describe the relationship between levels of self-criticism and dependence (DEQ) in patients with depression (BDI-I) and dropout of psychotherapy, exploring the mediating role of the therapeutic alliance (WAI-P) and the moderating role of patients' age. Using a longitudinal design and covenience sampling, 81 adult patients receiving brief psychotherapy were recruited at a private health center in Santiago, Chile. Twenty-two of these patients dropped out of treatment. Based on a regression model with interaction terms and controlling for patients' sex, schooling, and age and therapists' years of experience, results indicate that the higher the level of self-criticism the higher the probability of dropping out psychotherapy. This relationship is not explained by the therapeutic alliance. The association between self-criticism and drop out is not observed in younger patients (30 years), yet it is present in older patients (> 40 years). In addition, the higher the dependence, the lower the probability of dropping out of psychotherapy.

Augusto Mellado y Nicolás Suárez, Programa de Doctorado en Psicoterapia, Escuela de Psicología, Pontificia Universidad Católica de Chile, Santiago, Chile; Carola Pérez, Facultad de Psicología, Universidad del Desarrollo, Santiago, Chile; Paula Dagnino, Facultad de Psicología, Universidad Alberto Hurtado, Santiago, Chile; Sergio Gloger, Facultad de Medicina, Universidad de Chile, Santiago, Chile; Mariane Krause, Escuela de Psicología, Pontificia Universidad Católica de Chile, Santiago, Chile.

Este estudio recibió apoyo del Fondo de Innovación para la Competitividad del Ministerio de Economía, Fomento y Turismo de Chile, a través de la Iniciativa Científica Milenio, Proyecto IS130005, y del Programa de Cooperación Internacional de la Comisión Nacional de Investigación Científica y Tecnológica, a través del proyecto PII20150035.

La correspondencia relativa a este artículo debe ser dirigida a Augusto Mellado Mora, Programa de Doctorado en Psicoterapia, Escuela de Psicología, Pontificia Universidad Católica de Chile, Avda. Vicuña Mackenna 4860, Macul, Santiago, Chile. E-mail: apmellad@uc.cl 
Keywords: depression, self-criticism, dependence, therapeutic alliance, dropout in psychotherapy

La adherencia a una psicoterapia es uno de los primeros objetivos a lograr para obtener resultados significativos. Uno de los aspectos más problemáticos al momento de iniciar un proceso psicoterapéutico es la posibilidad de su finalización anticipada y abrupta por parte de los pacientes, de acuerdo con los criterios institucionales, de los psicoterapeutas y/o del modelo de psicoterapia utilizado.

Entre las razones que han motivado la investigación de este problema se encuentran el cuestionamiento a la efectividad y la relación costo-beneficio de las ofertas en salud mental de los servicios de salud (Garfield, 1986, citado en Wierzbicki \& Pekarik, 1993). En el ámbito clínico destacan las potenciales experiencias negativas que pudiesen haber gatillado un abandono y que podrían afectar futuras consultas y la formación de nuevos vínculos terapéuticos (Menéndez, Acosta, Bodon \& Mayorga, 2010).

Un meta-análisis (125 estudios) estimó en un 46,86\% el abandono de la psicoterapia, tanto al considerar pacientes adultos y niños con distintos diagnósticos, incluyendo terapias de diferentes modalidades (individuales, grupales, familiares y de pareja), orientación y duración. El tamaño del efecto promedio por sexo en las muestras de adultos $(M=-0,14)$ y mixtas $(M=0,04)$ fue menos extremo que en las muestras de niños $(M=-0,52)$, aunque en todos los casos las mujeres abandonaron más la terapia que los hombres (Wierzbicki \& Pekarik, 1993). Un segundo meta-análisis (73 estudios) estimó en un 35,26\% el abandono en pacientes adultos con distintos diagnósticos, en terapias individuales de distinta orientación y duración. El tamaño del efecto para el género fue $d_{\text {Cohen }}=0,18$ y las mujeres abandonaron más el tratamiento que los hombres (Sharf, 2008). Finalmente, un tercer meta-análisis estimó el abandono de pacientes adultos en un $19,7 \%$, tanto en terapias individuales como grupales. El tamaño del efecto para el género fue $d$ cohen $=0,01$, no encontrando diferencias entre mujeres y hombres respecto al abandono (Swift \& Greenberg, 2012).

Entre las características de quienes abandonaron la psicoterapia, se encuentran pertenecer a una minoría racial, un nivel educacional o socioeconómico bajo (Wierzbicki \& Pekarik, 1993) y ser más jóvenes (Sharf, 2008; Swift \& Greenberg, 2012). El abandono es más probable en pacientes diagnosticados con abuso de sustancias, psicosis y esquizofrenia (Hamilton, Moore, Crane \& Payne, 2011), cuando los pacientes no informan a sus terapeutas las inasistencias en las sesiones iniciales (Krebs, González, Rivera, Herrera \& Melis, 2012) y cuando presentan reducidas expectativas positivas sobre la psicoterapia, bajo nivel de autoeficacia y alta hostilidad e impulsividad (Sharf, 2008).

Un meta-análisis (587 estudios) que buscó determinar si existen diferencias en el abandono de pacientes adultos, dependiendo del modelo de tratamiento y de sus diagnósticos, encontró que en general el abandono no se diferencia según el modelo de psicoterapia, aunque sí hubo diferencias según el tipo de tratamiento en pacientes diagnosticados con depresión, trastornos de la conducta alimentaria y trastorno de estrés postraumático. Los tratamientos desarrollados desde el modelo integrativo tuvieron un porcentaje de deserción más bajo para depresión (10,9\%) y el trastorno de estrés postraumático $(8,8 \%)$, mientras que la terapia conductual dialéctica presentó un porcentaje más bajo de abandono (5,9\%) en pacientes con trastornos alimentarios (Swift \& Greenberg, 2014).

Si bien los antecedentes mencionados dan cuenta del abandono de la psicoterapia como un resultado negativo, también existen otros motivos que lo podrían explicar. En este sentido, el abandono puede producirse también debido a la sensación de mejoría o progreso por parte del paciente (Guzmán \& Mora, 1995; Ogrodniczuk, Joyce \& Piper, 2005). Puede entenderse, asimismo, como una acción de autocuidado de los pacientes en psicoterapias percibidas como dañinas o iatrogénicas (Porcel Medina, 2005) y puede presentarse en pacientes asertivos, independientes y buscadores activos de soluciones (Watson, 2011).

\section{Abandono de la Psicoterapia en Pacientes Diagnosticados con Depresión}

Dos meta-análisis sobre el abandono en psicoterapias individuales de adultos para el tratamiento de la depresión mayor y unipolar (54 y 23 estudios) reportaron un abandono promedio de 17,5\% y 24,63\%, respectivamente. El primero señala que el género $(b=0,303, p=0,16)$ no tuvo influencia en el abandono, a pesar de que las mujeres presentaron una probabilidad más elevada de abandonar la psicoterapia que los hombres, mientras que en el segundo se indica que la mayoría de los pacientes que finalizaron el tratamiento fueron mujeres $(M=68,61 \%, D E=9,57)$, en comparación con los hombres (Cooper \& Conklin, 2015; Hans \& Hiller, 2013). 
El abandono de la psicoterapia en pacientes adultos con depresión es más probable cuando los pacientes pertenecen a una minoría racial, son más jóvenes, presentan comorbilidades (p. ej., trastornos de personalidad, trastornos de ansiedad), mayor gravedad del cuadro al inicio del proceso, bajas expectativas positivas sobre la terapia, una reducida mejoría en las etapas iniciales y falta de farmacoterapia al comienzo (Arnow et al., 2007; Lopes, Gonçalves, Sinai \& Machado, 2015; Persons, Burns \& Perloff, 1988; Schindler, Hiller \& Witthöft, 2013; Simon \& Ludman, 2010).

Particularmente en Chile, un estudio sobre la efectividad y la adherencia del Programa para la Detección, Diagnóstico y Tratamiento Integral de la Depresión, puesto en marcha desde el 2001 por el Ministerio de Salud de Chile (MINSAL, 2001), indicó una tasa de abandono del 19,5\% en mujeres a los tres meses de tratamiento. A diferencia de lo encontrado en los estudios internacionales, las pacientes chilenas que abandonaron la terapia presentaron síntomas ansiosos y somatomorfos menos intensos, menores factores de riesgo psicosocial y menor satisfacción con la atención recibida (Alvarado, Vega, Sanhueza \& Muñoz, 2005).

\section{Rol de las Configuraciones Anaclítica e Introyectiva en el Desarrollo de la Personalidad y en la Comprensión y Tratamiento de la Depresión}

Blatt (2004, 2007, 2008) distingue dos dimensiones psicológicas fundamentales en el desarrollo de la personalidad: la relación interpersonal (interpersonal relatedness) y la autodefinición (self-definition). Ambas dimensiones estarían en interacción en un proceso continuo y dialéctico. La primera se refiere al desarrollo de relaciones interpersonales cada vez más maduras, íntimas y mutuamente satisfactorias y la segunda, al desarrollo de un sentido del yo o de identidad cada vez más diferenciado, integrado y realista. Sin embargo, es posible que por predisposiciones biológicas y/o eventos a mbientales altamente perturbadores se distorsione su integración y se llegue a un énfasis defensivo que exacerbe una dimensión a expensas de la otra. Así, se utiliza el término anaclítico para dar cuenta de la organización de la personalidad en la que predomina una inclinación exagerada hacia las relaciones interpersonales y el término introyectivo para dar cuenta de la organización de la personalidad en la que se exacerba la autodefinición.

Ambas distorsiones en el desarrollo de la personalidad podrían estar a la base de los distintos trastornos psicopatológicos (Luyten \& Blatt, 2015), más bien como reacciones desadaptativas a las demandas evolutivas de la personalidad que como categorías definidas por síntomas manifiestos. En la configuración anaclítica predominaría una preocupación exagerada por lograr relaciones interpersonales satisfactorias, descuidándose la evolución yoica y generando una fuerte dependencia hacia los demás, mientras que en la configuración introyectiva predominaría una preocupación exagerada por la autodefinición, a expensas de la construcción de relaciones interpersonales significativas (Blatt, 2004, 2007, 2008).

Blatt (2004, 2007, 2008) sugiere que a la base de los trastornos depresivos se encuentran estas dos configuraciones psicológicas distorsionadas. Se trataría de un trastorno no homogéneo que, aunque contempla un conjunto relativamente determinado de síntomas, se presenta y experimenta diferenciadamente en cada paciente (Blatt \& Luyten, 2009; Luyten \& Blatt, 2011). En la depresión de tipo anaclítico existiría una necesidad constante de establecer y mantener relaciones interpersonales íntimas satisfactorias y de dar y recibir afecto, buscando una sensación de protección, produciéndose, al mismo tiempo, una falta de diferenciación entre yo y los otros, en una interacción dependiente. Frente a la amenaza de las relaciones tenuemente construidas reaccionarían con defensas de tipo evitativo (p. ej., represión y retirada). En la depresión de tipo introyectivo (en la que se presenta una importante expresión autocrítica) existiría una necesidad de autonomía y control del propio cuerpo y mente, buscando constantemente el valor propio y una identidad integrada. Se trataría de personas racionales en las que sobresalen en sus conflictos la rabia y la agresión hacia sí mismos y hacia los demás, además de importantes sentimientos de culpa. De esta manera, se afectarían sus relaciones interpersonales por las disputas por la mantención de un sentido viable para el yo. Los conflictos suelen ser tratados de manera parcial y disfrazados mediante defensas de tipo reactivo (p. ej., aislamiento e intelectualización).

Las características de ambas configuraciones subyacentes a la depresión han sido estudiadas y los resultados sugieren no solo la idoneidad de un diagnóstico diferenciado para cada caso, sino también una forma particular de abordaje terapéutico (Coyne \& Whiffen, 1995; Luthar \& Blatt, 1995; Luyten et al., 2007; Shahar, 2006; Shahar, Joiner Jr., Zuroff \& Blatt, 2004; Zuroff \& Mongrain, 1987). Respecto de la forma en que se presentan estas diferencias en la psicoterapia, los pacientes depresivos anaclíticos responderían mejor a un contexto terapéutico basado en una relación interpersonal cálida con su terapeuta, mientras que los 
pacientes depresivos introyectivos responderían mejor a un contexto terapéutico basado en su autonomía, independencia y en propuestas ideacionales (Blatt, Shahar \& Zuroff, 2001). Otra arista identificada en relación a la psicoterapia es la representación que los pacientes hacen de sí mismos y de los demás, al comenzar algún proceso psicoterapéutico. Aquellos con depresión anaclítica se auto-representarán en un estado de necesidad de los otros significativos y a los otros los representarán como ausentes, descuidados y abandonadores, mientras que los pacientes con depresión introyectiva se auto-representarán como no dignos y a los otros, como intrusivos, controladores y castigadores (Blatt, 1974; Blatt, Wein, Chevron \& Quinlan, 1979, citado en Blatt, Zuroff, Hawley \& Auerbach, 2010).

El presente estudio se centró en la dimensión del autocriticismo, propio de la configuración introyectiva, ya que estos pacientes tienen dificultades para comprometerse en la relación terapéutica, necesitando más tiempo para lograr una alianza suficientemente buena (Luyten, Corveleyn \& Blatt, 2005). En las psicoterapias breves el hecho de no contar con el tiempo suficiente para construir una relación basada en la confianza puede influenciar su abandono.

\section{Autocriticismo como Aspecto Fundamental de la Depresión}

La investigación en depresión ha puesto énfasis en el autocriticismo y el perfeccionismo (en su carácter no adaptativo), como aspectos de la personalidad que influyen significativamente en su aparición y curso, sugiriéndose como un elemento clave que permite comprender su vulnerabilidad y sus manifestaciones más problemáticas (Campos, Besser \& Blatt, 2010; Dunkley, Sanislow, Grilo \& McGlashan, 2006, 2009; Enns \& Cox, 1999; Hewitt \& Flett, 1991). Si bien autocriticismo y perfeccionismo podrían presentar algunas diferencias para la comprensión de la depresión, las investigaciones suelen tratarlos como términos similares, definiendo al perfeccionismo orientado al sí mismo como "la tendencia a considerar el self en términos críticos" (Shahar, Blatt, Zuroff, Krupnick \& Sotsky, 2004, p. 142), o "relacionado con altos estándares de logro y autocriticismo por no satisfacerlos" (Egan, Wade \& Shafran, 2011, p. 204). Incluso, se ha señalado que las psicopatologías introyectivas involucran un alto grado de autocriticismo y perfeccionismo (Blatt, 1995). Los individuos perfeccionistas vivirían la depresión focalizada en temas relacionados con la auto-valoración y el autocontrol, criticándose y atacándose a sí mismos, y en presencia de sentimientos intensos de culpa, vergüenza, de fallar y autodevaluarse, sustentándose esta forma de experiencia en el riesgo a fallar, al encontrarse durante su infancia con normas parentales severamente impuestas (Blatt, 1995). Algunas de las dimensiones del perfeccionismo asociadas a la presencia de psicopatología depresiva serían la presión que estos individuos perciben desde los otros para conseguir logros (o perfeccionismo socialmente prescrito) y una autoevaluación negativa frente a los errores o al intento de conseguir tales logros (DiBartolo, Li \& Frost, 2008; Enns \& Cox, 1999). También se ha encontrado que los individuos que tienen pensamientos perfeccionistas frecuentes presentan tendencias desadaptativas de regulación racional-emotiva y altos niveles de depresión (Rudolph, Flett \& Hewitt, 2007).

Un estudio realizado en una muestra de 43 pacientes adultos chilenos con depresión identificó una correlación positiva entre su nivel de autocriticismo y la gravedad de su sintomatología depresiva, siendo este efecto más marcado en aquellos pacientes que presentan una baja integración en la función estructural de autorregulación, en comparación con quienes presentan una autorregulación integrada (Dagnino, Pérez, Gómez, Gloger \& Krause, 2017).

\section{Relación entre Autocriticismo y Efectividad en la Psicoterapia de la Depresión}

Los estudios en este campo evidencian que altos niveles de autocriticismo pueden impedir resultados gananciales en psicoterapias breves, debido a la dificultad de estos pacientes para involucrarse con sus terapeutas en un tiempo acotado, la experimentación de sensaciones de insuficiencia y desilusión hacia el tratamiento, la dificultad para establecer y mantener relaciones sociales y posibles proyecciones de esquemas relacionales exigentes y frustrantes (Blatt, 1995; Blatt, Quinlan, Pilkonis \& Shea, 1995; Blatt, Zuroff, Bondi, Sanislow III \& Pilkonis, 1998; Egan et al., 2011; Shahar, Blatt et al., 2004; Shahar, Blatt \& Zuroff, 2007). Más específicamente, Blatt et al. (1998) han sugerido que estos pacientes presentan dificultades para obtener ganancias terapéuticas a partir de la última mitad del tratamiento, posiblemente por su disconformidad al sentir que la finalización de la terapia es unilateral, limitada y arbitrariamente impuesta. Además, estos resultados deficitarios no solo afectarían el periodo de tratamiento, sino que se extenderían post tratamiento, perjudicando, entre otras cosas, la habilidad de estos pacientes para afrontar el estrés (Blatt \& Zuroff, 2005). 
Blatt $(1995,2007)$ puntualiza que, si bien estos pacientes podrían no obtener beneficios significativos en terapias breves, sí logran mejores resultados que otros grupos de pacientes (con depresiones anaclíticas) en psicoterapias psicodinámicamente orientadas y de larga duración, lo que sugiere que necesitan mayor tiempo para adecuarse a una relación terapéutica y comenzar a cambiar representaciones negativas yoicas arraigadas, mediante una actividad terapéutica que promueva sus procesos asociativos y disminuya su distanciamiento interpersonal.

\section{Relación entre Autocriticismo, Alianza Terapéutica y Resultados Terapéuticos}

Estudios que evaluaron la relación entre autocriticismo y alianza terapéutica encontraron que el autocriticismo estuvo asociado con bajos niveles en la alianza percibida por los pacientes (Egan et al., 2011; Whelton, Paulson \& Marusiak, 2007). Coherente con ello, Zuroff et al. (2000) reportan que la contribución de los pacientes a la alianza se incrementó constantemente a lo largo del tratamiento entre pacientes con bajos niveles de perfeccionismo, mientras que en pacientes con altos niveles de perfeccionismo este aumento fue mínimo o ausente. Estos autores presentan un modelo mediacional en el cual la relación inversa entre perfeccionismo y resultados terapéuticos fue parcialmente explicada por la dificultad de los pacientes que puntuaron un mayor nivel de perfeccionismo para desarrollar una alianza terapéutica más fuerte — variable mediadora-durante el transcurso de la terapia.

Sin embargo, no todos los estudios llegan a las mismas conclusiones. En una investigación con pacientes que cursaron terapias breves para la depresión se reportó que una positiva relación terapéutica inicial (que contribuye a la alianza terapéutica) predice mejores resultados y el mejoramiento de capacidades adaptativas, independientemente de algunas características personales de los pacientes, entre las que se incluyó el perfeccionismo y la necesidad de aprobación (Zuroff \& Blatt, 2006).

Finalmente, un estudio longitudinal mostró que una alianza terapéutica fuertemente establecida se correlaciona inversamente con el grado de perfeccionismo de los pacientes depresivos y que el perfeccionismo se correlaciona positivamente con la sintomatología de los pacientes a lo largo de la psicoterapia (Hawley, Ho, Zuroff \& Blatt, 2006).

\section{Alianza Terapéutica y Abandono en Psicoterapia}

Ahora bien, al abordar específicamente la relación entre alianza terapéutica y abandono, un meta-análisis (11 estudios) indicó una asociación inversa y moderadamente fuerte entre ambas variables, siendo más probable que los pacientes que presentaron una alianza terapéutica más débil abandonaran la psicoterapia. Además, indica que hay variables que moderan esta relación entre alianza y abandono de la terapia, a saber, en los pacientes de mayor nivel educacional la asociación entre alianza y abandono es más débil y, por su parte, en las psicoterapias de mayor duración dicha asociación es más marcada (Sharf, Primavera \& Diener, 2010).

En síntesis, se han reportado diversas variables relacionadas con el abandono en psicoterapia y particularmente con el abandono en la psicoterapia de la depresión. Sin embargo, parece no haber claridad respecto a cuáles podrían ser aquellas más significativas para la comprensión de esta problemática. Una de las relaciones destacada en los estudios internacionales es entre la intensidad de los síntomas ansiosos y el abandono (Cooper \& Conklin, 2015; Hans \& Hiller, 2013), aunque uno de los pocos estudios realizados en Chile (Alvarado et al., 2005) encontró el resultado inverso. Es decir, la intensidad de los síntomas ansiosos no debe ser considerada siempre como una condición que lleve a los pacientes depresivos a no continuar su psicoterapia.

Por otra parte, en estos estudios los aspectos caracterológicos de los pacientes quedan vagamente definidos. Aparentemente, no es posible identificar un aspecto caracterológico específico asociado al abandono, más aún en las psicoterapias de la depresión, en las que precisamente la comorbilidad con trastornos de la personalidad es habitual (Arnow et al., 2007; Cooper \& Conklin, 2015; Hans \& Hiller, 2013; Lopes et al., 2015; Persons et al., 1988; Schindler et al., 2013; Simon \& Ludman, 2010). Considerando esto último, puede evaluarse si el autocriticismo, una de las configuraciones más comunes y problemáticas de la depresión, podría tener alguna relación con el abandono en psicoterapia en el contexto chileno. Si bien las investigaciones revisadas sugieren que los pacientes autocríticos tienen dificultades para conseguir buenos resultados en psicoterapias breves para el tratamiento de la depresión (que son las más utilizadas en el sistema de salud chileno), no establecen si estos pobres resultados pudieron gatillar abandonos. En contraste, 
los pacientes que presentan características dependientes, propias de la configuración anaclítica, podrían permanecer en psicoterapia, debido a su búsqueda de apoyo en los demás (Blatt et al., 1994).

Un último aspecto de importancia es la eventual influencia que podría tener la alianza terapéutica en esta aparente tensión entre autocriticismo y la decisión de continuar una psicoterapia, ya que se ha señalado que la alianza resulta un elemento importante para la prevención del abandono (Sharf et al., 2010), aunque también se deja en claro que los pacientes que presentan altos niveles de perfeccionismo tienen problemas en la construcción de la alianza durante distintas etapas de su tratamiento (Egan et al., 2011; Whelton et al., 2007; Zuroff \& Blatt, 2006; Zuroff et al., 2000).

Considerando todo lo anterior, este estudio tuvo como propósito principal describir la relación entre el nivel de autocriticismo en pacientes con depresión y el abandono de la psicoterapia, evaluando el rol mediador de la alianza terapéutica inicial (sesión 3) desde la perspectiva de los pacientes. En consecuencia, se hipotetizó que: (a) el nivel de autocriticismo al inicio de la psicoterapia en los pacientes con depresión predice el abandono durante el proceso psicoterapéutico y (b) la alianza terapéutica establecida en las primeras sesiones de la psicoterapia media la relación entre el autocriticismo de los pacientes y el abandono de su psicoterapia. Finalmente, se exploró un potencial efecto moderador entre las variables en estudio, incluyendo variables sociodemográficas y la variable dependencia, puesto que podría ser un factor protector del abandono.

\section{Método}

Este estudio comenzó siendo parte de la línea de investigación "Procesos de cambio en depresión" del Núcleo Milenio de Intervención Psicológica y Cambio en Depresión, alojadas en la Escuela de Psicología de la Pontificia Universidad Católica de Chile. Posteriormente, se integró al Instituto Milenio para el Estudio de la Depresión y la Personalidad.

\section{Participantes}

Los participantes fueron pacientes adultos diagnosticados con depresión y atendidos en psicoterapia para la depresión en un centro de salud privado en Santiago de Chile. Los pacientes fueron invitados inicialmente vía telefónica, mediante una consigna creada por el equipo de investigación que fue transmitida por el personal administrativo del centro de salud cuando eran citados a la primera sesión de psicoterapia. La consigna consistía en ofrecerles participar voluntariamente en un estudio sobre psicoterapia de la Pontificia Universidad Católica de Chile y se los citaba una hora antes de su primera sesión para reunirse con un integrante del equipo de investigación, quien explicaba en detalle en qué consistía el estudio, las condiciones para su desarrollo (como la participación voluntaria, su independencia respecto a la psicoterapia y la confidencialidad en el manejo de los datos recolectados) y, si correspondía, iniciaba el proceso de consentimiento informado.

La muestra, obtenida por conveniencia, estuvo constituida por 81 pacientes adultos con un promedio de edad de 43,4 años $(D E=13,35)$, de los cuales 22 (27\%) abandonaron la psicoterapia.

Las características de los pacientes participantes pueden observarse en la Tabla 1.

\section{Instrumentos y Mediciones}

Inventario de Depresión de Beck (BDI-I; Beck, Ward, Mendelson, Mock \& Erbaugh, 1961). El BDI-I es un instrumento de autorreporte que se utiliza para evaluar el nivel de sintomatología depresiva. Se utilizó la versión BDI-IA, que consta de 21 ítems con cuatro opciones de respuesta cada uno (0 a 3 puntos), donde un mayor puntaje indica mayor sintomatología depresiva (0-63 puntos). Por ejemplo, en el ítem referido a tristeza, el paciente debe indicar si No se siente triste (0), Se siente triste (1), Se siente triste continuamente y no puede dejar de estarlo (2) o Ya no puede soportar su pena (3). El instrumento define cuatro categorías diagnósticas para depresión: mínima (0-9), leve (10-18), moderada (19-29) y severa (30-63). Datos psicométricos en muestras chilenas sugieren una estructura unifactorial (obtenida a través de un análisis factorial exploratorio y corroborada por un análisis factorial confirmatorio) y una consistencia interna, evaluada con alfa de Cronbach, de $\alpha=0,92$ (Valdés et al., 2017). En el presente estudio, la consistencia interna fue de $\alpha=0,86$. 
Tabla 1

Características de los Participantes del Estudio

\begin{tabular}{|c|c|c|}
\hline Variable & $\begin{array}{c}\text { Pacientes que terminan la } \\
\text { psicoterapia }(N=59)\end{array}$ & $\begin{array}{c}\text { Pacientes que abandonan la } \\
\text { psicoterapia }(N=22)\end{array}$ \\
\hline Sexo (mujeres) & $52(88,1 \%)$ & $17(77,3 \%)$ \\
\hline Edad promedio & $47,76(D E=11,61$, Rango 19-66) & $31,86(D E=10,67$, Rango $19-57)$ \\
\hline \multicolumn{3}{|l|}{ Escolaridad } \\
\hline Básica incompleta & $1(1,7 \%)$ & $0(0,0 \%)$ \\
\hline Básica completa & $4(6,8 \%)$ & $0(0,0 \%)$ \\
\hline Media incompleta & $6(10,1 \%)$ & $3(13,6 \%)$ \\
\hline Media completa & $15(25,4 \%)$ & $4(18,2 \%)$ \\
\hline Superior técnico-profesional & $10(16,9 \%)$ & $5(22,7 \%)$ \\
\hline Superior universitaria & $23(39,0 \%)$ & $10(45,5 \%)$ \\
\hline Sintomatología depresiva ${ }^{a}$ & $23,84(D E=9,37$, Rango 5-41) & $29,62(D E=9,06$, Rango $12-46)$ \\
\hline Sesiones & $10,03(D E=4,22$, Rango 5-21) & $9,82(D E=8,94$, Rango 3-41) \\
\hline Experiencia del terapeuta ${ }^{b}$ & $8,54(D E=4,55$, Rango $4-17)$ & $9,59(D E=5,27$, Rango 4-25) \\
\hline En tratamiento farmacológico ${ }^{c}$ & $56(94,9 \%)$ & $20(90,9 \%)$ \\
\hline
\end{tabular}

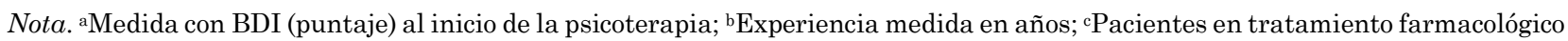
en forma paralela al proceso psicoterapéutico.

Cuestionario de Experiencias Depresivas (DEQ; Blatt, D'Afflitti \& Quinlan, 1976, 1979; Zuroff, Quinlan \& Blatt, 1990). Este cuestionario de autorreporte se utilizó para evaluar el nivel de autocriticismo y dependencia en la personalidad. Consta de 66 ítems, presentados en una escala tipo Likert de 7 puntos $(1=$ totalmente en desacuerdo, $7=$ totalmente de acuerdo). Cada uno de ellos aporta diferencialmente a la estimación de los puntajes de los factores autocriticismo (p. ej., "A menudo me siento culpable"), dependencia (p. ej., "Soy muy sensible a las señales de rechazo de los otros") y autoeficacia (p. ej., "Tengo muchos recursos internos"), al ser multiplicados por coeficientes factoriales obtenidos de la muestra original (Blatt et al., 1976). Elevados puntajes indican mayor presencia de la dimensión. Para este estudio se utilizó una versión traducida al español para el contexto chileno (Rost \& Dagnino, 2011), la cual se encuentra en proceso de adaptación para población consultante en Chile (J. C. Pérez, comunicación personal, Septiembre 25, 2018).

La estructura factorial de la escala original fue obtenida a través de análisis de componentes principales en una muestra no clínica de estudiantes universitarios (rotación Varimax), los cuales mostraron validez convergente con reporte de ánimo, preocupación por la muerte y diferencial entre el self-real versus ideal (Blatt et al., 1976). Se ha encontrado una confiabilidad test-retest muy alta para autocriticismo $(r=0,75) \mathrm{y}$ dependencia ( $r=0,81$; Zuroff, Moskowitz, Wielgus, Powers \& Franko, 1983), demostrando estabilidad en el tiempo. La consistencia interna reportada por Blatt, Quinlan, Chevron, McDonald \& Zuroff (1982) y evaluada por alfa de Cronbach es $\alpha=0,81$ para la escala de dependencia y $a=0,80$ para la escala de autocriticismo, basándose en la selección de los cinco ítems de mayor carga factorial para cada caso. Siguiendo el mismo procedimiento, en la presente muestra la consistencia interna fue de $\alpha=0,49$ para la escala de dependencia y de $\alpha=0,75$ para la escala de autocriticismo.

Inventario de Alianza de Trabajo-versión Paciente (WAI-P; Horvath \& Greenberg, 1989). Es un instrumento de autorreporte que se utiliza para evaluar la alianza terapéutica desde la perspectiva de los pacientes, a partir de tres dimensiones: la calidad del vínculo afectivo entre paciente y terapeuta (p. ej., "Creo que [el terapeuta] me estima"), sus acuerdos en metas (p. ej., "Las metas de estas sesiones son importantes para mí") y sus acuerdos en tareas (p. ej., "Creo que [el terapeuta] y yo estamos de acuerdo sobre lo que para mí es importante trabajar"). Consta de 36 ítems, respondidos en una escala tipo Likert de 7 puntos ( $1=$ nunca, 7 = siempre). Cada una de las subescalas está conformada por 12 ítems; a mayor puntaje mejor calidad de alianza. Se utilizó la versión adaptada para Chile, cuyo análisis factorial identificó un solo factor, que explica el $82,5 \%$ de la varianza (mostrando altas correlaciones entre las tres subescalas), y que presenta una adecuada consistencia interna de $\alpha=0,91$ (Santibáñez, 2003). En el presente estudio la consistencia interna fue de $\alpha=0,68$. 
Ficha de datos sociodemográficos. Incluye datos generales de identificación de los pacientes, tales como edad, fecha de nacimiento, estado civil actual, ocupación actual, escolaridad y tratamiento farmacológico.

Abandono de la psicoterapia. El criterio para definir el abandono de la psicoterapia, denominado por la institución alta administrativa, fue la deserción de los pacientes del proceso psicoterapéutico antes de que los terapeutas tratantes indicaran el alta clínica, es decir, antes de que se considerara finalizada la psicoterapia por parte de los especialistas y por el protocolo fijado por la institución tratante. Se consideraron abandonos en la medida que los pacientes no dieron aviso de su deserción (la institución considera que el alta administrativa se da una vez que el paciente tiene hora agendada y suspende o falta en dos ocasiones seguidas) y que no volvieron a contactarse posteriormente con el terapeuta tratante para retomar la psicoterapia.

\section{Procedimiento}

Quienes aceptaron voluntariamente participar del estudio leyeron y firmaron un consentimiento informado aprobado por el comité de ética del centro de salud, además del comité de ética de la Escuela de Psicología de la Pontificia Universidad Católica de Chile. Los pacientes no recibieron ningún tipo de incentivo económico o material por su colaboración en el estudio y no hubo registro de los pacientes que rechazaron participar. Durante el desarrollo del estudio, nueve participantes decidieron retirarse sin explicitar razones y conforme a lo señalado en el consentimiento informado.

La aplicación de los instrumentos fue realizada por estudiantes del Programa de Doctorado en Psicoterapia y del Magister en Psicología Clínica de la Pontificia Universidad Católica de Chile, efectuándose periódicamente reuniones de seguimiento con los responsables del estudio respecto del trabajo efectuado. Los instrumentos fueron aplicados en el centro de salud en el que los pacientes recibieron su tratamiento psicológico.

Los pacientes completaron una ficha con sus datos sociodemográficos y respondieron el DEQ y el BDI-IA. El IAT-P fue completado al finalizar la tercera sesión, ya que los estudios sugieren que la alianza evaluada en esta etapa se relaciona positivamente con los resultados posteriores (Horvath \& Symonds, 1991). Además, puntajes bajos de la alianza en la tercera sesión, en comparación con la alianza conseguida en el resto del proceso, se asocian al abandono de la psicoterapia (Botella \& Corbella, 2011; Tryon \& Kane, 1995).

Las psicoterapias fueron realizadas según el procedimiento del centro de salud (IPGES; nombre ficticio), que es una institución privada de salud mental prestadora del Régimen General de Garantías Explícitas en Salud (GES). Los pacientes son derivados a psicoterapia una vez que han sido diagnosticados (criterios CIE-10) con depresión (leve, moderada o severa) por los psiquiatras de la institución. Los psicoterapeutas tienen entrenamiento de postítulo acreditado en distintos modelos (psicoanalítico, sistémico o integrativo). Su objetivo es tratar en un número acotado de sesiones la sintomatología depresiva. Pueden integrar diversas intervenciones eficaces para disminuir síntomas, mejorar el ajuste social y minimizar posibilidades de recaídas. El psicoterapeuta decide de manera autónoma qué procedimientos utiliza para lograr dicho objetivo, con tiempos de duración estandarizados por severidad (6 sesiones para la depresión leve, 8 sesiones para la moderada y 12 sesiones para la severa), acorde con la guía clínica del MINSAL. El criterio usado por el psicoterapeuta para establecer el alta clínica considera el logro de los objetivos específicos definidos para determinado episodio depresivo y la respuesta sintomática del paciente. El paciente está en conocimiento de que se busca lograr los resultados terapéuticos en esa cantidad de sesiones. El psicoterapeuta puede, considerando el protocolo institucional que incluye su valoración clínica y los resultados del Cuestionario sobre la Salud del Paciente (PHQ9), elevar una solicitud especial para aumentar ese número de sesiones a fin de lograr el objetivo terapéutico (hasta por un 50\% adicional de sesiones asignadas inicialmente o más en algunos casos).

Las psicoterapias tuvieron un promedio de 9,89 sesiones $(D E=5,9)$. Los terapeutas tratantes, hombres y mujeres, tenían en promedio 36,44 $(D E=6,72)$ años de edad y 8,57 $(D E=4,41)$ años de experiencia clínica.

\section{Análisis de Datos}

Se estimaron los datos descriptivos (promedios y $D E$ para las variables continuas y porcentaje para las categóricas) y correlaciones de las variables en estudio. Para evaluar la homogeniedad entre los dos grupos 
(pacientes que terminan la terapia y quienes abandonan) se utilizaron pruebas $t$ de Student y $\chi^{2}$ de Pearson. Cada vez que fue necesario, se realizó un cálculo del tamaño del efecto a través del índice $d$ de Cohen.

A continuación se presentan en detalle los procedimientos usados para el estudio de los modelos de mediación y moderación.

Modelo de mediación. Se estimó un modelo mediacional, utilizando regresión múltiple, para evaluar la hipótesis de mediación entre el autocriticismo de los pacientes (X) y la probabilidad de abandono de la psicoterapia (Y), considerando la alianza del paciente en la tercera sesión como variable mediadora (M). Adicionalmente, se incluyeron sexo y edad de los pacientes y experiencia del terapeuta como variables de control. Siguiendo lo estipulado por Hayes (2013), quien establece que los modelos mediacionales no imponen la exigencia de probar en forma previa la asociación simple entre las variables $\mathrm{X}$ e $\mathrm{Y}$, se llevó a cabo el siguiente procedimiento:

Cada modelo implicó la estimación de dos ecuaciones de regresión simultáneas, usando la macro sintáxis denominada PROCESS, desarrollada por Andrew F. Hayes (disponible en www.afhayes.com) para el programa SPSS-v21. Cuando se consideran variables dependientes dicotómicas, la macro sintáxis estima regresiones logísticas.

(a) La variable mediadora alianza (M) fue regresada en función del autocriticismo de los pacientes:

$$
\mathrm{M}=i_{1}+a * \text { Autocriticismo }+B_{1} * \text { Sexo-Pcte }+B_{2} * \text { Edad-Pcte }+B_{3} * \text { Experiencia-Tpta }
$$

(b) La variable criterio probabilidad de abandono (Y) fue regresada en función de la variable mediadora alianza, controlando el efecto del autocriticismo y de las variables sociodemográficas (sexo y edad del paciente) y experiencia del terapeuta:

$$
\mathrm{Y}=i_{2}+b * \text { Alianza }+c^{\prime} * \text { Autocriticismo }+B_{1} * \text { Sexo-Pcte }+B_{2} * \text { Edad-Pcte }+B_{3} * \text { Experiencia-Tpta }
$$

En estas ecuaciones $\mathrm{M}$ es la variable mediadora alianza terapéutica e Y es la variable probabilidad de abandono, $a$ es el coeficiente de regresión que da cuenta de cuánto predice el abandono la variable mediadora alianza terapéutica, ajustado por las variables sociodemográficas (sexo y edad del paciente) y experiencia del terapeuta, e $i$ es el intercepto de la ecuación de regresión. El parámetro c'relaciona el predictor autocriticismo con la probabilidad de abandono, ajustado por la variable mediadora y las variables control; $b$ es el coeficiente de regresión que relaciona la variable mediadora con la probabilidad de abandono, controlando el autocriticismo y las variables sociodemográficas y experiencia del terapeuta. $B_{i}$ corresponde a los coeficientes de regresión de las variables control.

En una segunda etapa se estiman los parámetros del efecto indirecto (que da cuenta del proceso de mediación), a través de la estimación del producto de los parámetros a x b. Para determinar si este efecto indirecto es significativo, se utilizó el método bootstrapping, que consiste en extraer múltiples muestras de los datos y estimar los efectos indirectos ( $\mathrm{a}$ x b) en cada una de estas muestras. En este estudio se utilizó el método bootstrapping presentando los intervalos de confianza (95\% IC) con 1.000 muestras. Se construye así una aproximación empírica de la distribución muestral a x b, la que se utiliza para construir intervalos de confianza para el efecto indirecto o mediado. En la medida que este intervalo no incluya el valor cero se estima que hay efecto mediado significativo.

Moderación. Se estimó un modelo de regresión con interacción. Se ingresaron, en primer lugar, las características demográficas de los pacientes, edad, sexo y escolaridad de los pacientes, y la experiencia de los terapeutas (variables de control), posteriormente se ingresaron los predictores de primer orden (autocriticismo, dependencia) y las interacciones de primer orden (autocriticismo x edad de los pacientes). Las interacciones entre las variables demográficas y del estudio fueron estimadas en forma exploratoria, particularmente respecto de la edad, ya que la evidencia señala que los pacientes más jovenes tienden a abandonar con mayor regularidad la psicoterapia (Arnow et al., 2007; Sharf, 2008; Simon \& Ludman, 2010; Swift \& Greenberg, 2012). Se evaluó el nivel de significación de los coeficientes de regresión de los componentes de interacción estimados, manteniendo en el modelo solo aquellas interacciones significativas. Dado que se presentó una interacción entre autocriticismo $\mathrm{x}$ edad de los pacientes, se estimaron tres ecuaciones de regresión parcial, que daban cuenta de la asociación entre autocriticismo y probabilidad de abandono en tres niveles del predictor edad: alto (1 $D E$ sobre el promedio), medio (promedio) y bajo (1 $D E$ bajo el promedio). Dichas ecuaciones fueron graficadas. Para ello se utilizó el macro PROCESS, desarrollado por Andrew F. Hayes. 


\section{Resultados}

\section{Diferencia entre los Grupos}

Respecto de la homogeneidad entre el grupo de pacientes que terminan su tratamiento y quienes abandonan, en cada una de las variables estudiadas se encontraron los siguientes resultados: el nivel de autocriticismo fue mayor en quienes abandonaron la terapia $(M=0,95, D E=0,90, n=22)$ que en quienes permanecieron $(M=0,31, D E=0,81, n=59), t(79)=3,09, p=0,003,95 \%$ IC $[0,22,1,05], d$ cohen $=0,75$; en el nivel de dependencia no hubo diferencias entre ambos grupos, presentando quienes abandonaron un promedio de $-0,26$ puntos $(D E=0,95, n=22)$ y los que permanecieron, un promedio de 0,09 puntos $(D E=0,92, n=59), t(79)=-1,51, p=0,135$; el nivel de alianza fue mayor en quienes permanecieron $(M=5,41$, $D E=0,09, n=59)$ que en quienes abandonaron $(M=5,34, D E=0,15, n=22), t(79)=-2,44, p=0,017$, $95 \%$ IC $[-0,12,-0,01], d_{\text {Cohen }}=0,54$; los que abandonaron presentaron una mayor sintomatología depresiva $(M=29,62, D E=9,07, n=21)$ que quienes permanecieron $(M=23,85, D E=9,37, n=57), t(76)=2,43$, $p=0,017,95 \%$ IC $[1,05,10,50], d_{\text {Cohen }}=0,62$; quienes completaron la terapia presentaron un promedio de edad mayor $(M=47,76, D E=11,61, n=59)$ que quienes la abandonaron $(M=31,86, D E=10,67, n=22)$, $t(79)=-5,59, p<0,001,95 \%$ IC $[-21,55,-10,24], d$ cohen $=1,43$; la experiencia del terapeuta no mostró diferencias entre ambos grupos, presentando para el grupo de quienes abandonan un promedio de 9,59 años $(D E=5,27, n=22)$ y para quienes permanecieron un promedio de 8,54 años $(D E=4,55, n=59), t(79)=1,28$, $p=0,204$; tampoco hubo diferencias en el número de sesiones realizadas para cada caso, $t(79)=-0,11$, $p=0,914$; no hubo diferencias respecto al sexo del paciente en cada grupo, $\chi^{2}(1, N=81)=1,49, p=0,221$ [en una casilla hubo una frecuencia esperada menor que 5]; no hubo diferencia respecto de su escolaridad, $\chi^{2}(5, N=81)=2,85, p=0,723$ [en seis casillas hubo una frecuencia esperada menor que 5] ni en cuanto al tratamiento farmacológico actual, $\chi^{2}(1, N=80)=1,06, p=0,301$ [dos casillas presentaron una frecuencia esperada menor que 5].

\section{Correlaciones entre Variables}

Se obtuvo una matriz de correlaciones bivariadas y parciales entre las variables del estudio (ver Tabla 2), estas últimas controlando las variables sexo del paciente (se han descrito altos niveles de autocriticismo en los hombres), escolaridad del paciente (por la influencia ya descrita para el abandono) y años de experiencia del psicoterapeuta (eventual diferencia del manejo clínico). Se destacan aquellas correlaciones parciales positivas entre las variables autocriticismo y abandono de la psicoterapia, $r(81)=0,40, p<0,001$, autocriticismo y sintomatología depresiva, $r(81)=0,53, p<0,001$, además de las correlaciones parciales inversas entre autocriticismo y alianza percibida por los pacientes en la tercera sesión, $r(81)=-0,24$, $p=0,035$, y autocriticismo y edad de los pacientes, $r(81)=-0,36, p=0,001$. Del mismo modo, se encontró una correlación parcial positiva entre sintomatología depresiva y abandono de la psicoterapia, $r(81)=0,26$, $p=0,023$, y una correlación parcial inversa entre edad del paciente y abandono de la psicoterapia $r(81)=-0,51$, $p<0,001$, y entre alianza percibida por los pacientes en la tercera sesión y abandono de la psicoterapia, $r(81)=-0,24, p=0,040$.

\section{Relación entre Autocriticismo y Abandono Mediada por la Alianza Terapéutica}

Para evaluar la mediación de la alianza percibida por los pacientes en la tercera sesión, entre el nivel de autocriticismo y el abandono de la psicoterapia, se utilizó el método de evaluación de efectos indirectos (Preacher \& Hayes, 2008). El modelo se estimó controlando las variables sexo de los pacientes, edad de los pacientes y años de experiencia del terapeuta. Sus resultados se presentan en la Tabla 3 y muestran que la relación entre autocriticismo y alianza (efecto a) no fue significativa, $b=-0,01, p=0,20$, y que tampoco lo fue la relación entre alianza y abandono de la psicoterapia (efecto b), $b=-12,73, p=0,08$. Concordante con ello, la mediación propuesta no fue significativa, dado que el efecto mediado (a $\mathrm{x} \mathrm{b})=0,11(E E=0,12)$, $95 \%$ IC [-0,62, 0,12], es decir, la relación positiva entre autocriticismo y abandono no es explicada por la alianza terapéutica establecida en la tercera sesión. 
Tabla 2

Correlaciones entre las Variables del Estudio

\begin{tabular}{|c|c|c|c|c|c|c|c|c|c|c|c|c|}
\hline & Variable & $M$ & $D E$ & 1 & 2 & 3 & 4 & 5 & 6 & 7 & 8 & 9 \\
\hline 1. & Autocriticismo & 0,48 & 0,88 & & $0,313^{* *}$ & $0,328^{* *}$ & $-0,176$ & $0,482^{* *}$ & $-0,298 * *$ & $-0,103$ & $-0,230^{*}$ & $-0,049$ \\
\hline 2. & Dependencia & 0,00 & 0,94 & $0,373^{* *}$ & & $-0,168$ & $-0,025$ & 0,177 & 0,000 & 0,141 & 0,164 & $-0,131$ \\
\hline 3. & Abandono & - & - & $0,400 * *$ & $-0,134$ & & $-0,266^{*}$ & $0,269^{*}$ & $-0,533^{* *}$ & $-0,136$ & 0,117 & 0,143 \\
\hline 4. & Alianza terapéutica ${ }^{a}$ & 5,39 & 0,12 & $-0,243^{*}$ & $-0,060$ & $-0,238^{*}$ & & $-0,165$ & 0,108 & 0,011 & $-0,091$ & 0,171 \\
\hline 5. & Sintomatología depresiva & 25,40 & 9,59 & $0,527 * *$ & 0,217 & $0,262^{*}$ & $-0,214$ & & $-0,256^{*}$ & 0,065 & $-0,053$ & 0,220 \\
\hline 6. & Edad del paciente & 43,44 & 13,36 & $-0,362^{* *}$ & $-0,022$ & $-0,512^{* *}$ & 0,041 & $-0,257^{*}$ & & 0,014 & $-0,107$ & $-0,056$ \\
\hline 7. & Sexo del paciente & - & - & - & - & - & - & - & - & & 0,055 & $-0,112$ \\
\hline 8. & Escolaridad del paciente & - & - & - & - & - & - & - & - & - & & $-0,086$ \\
\hline 9. & Experiencia del terapeuta & 8,57 & 4,41 & - & - & - & - & - & - & - & - & \\
\hline
\end{tabular}

Nota. Las correlaciones bivariadas se presentan sobre la diagonal y las correlaciones parciales controlando el sexo y escolaridad del paciente y experiencia del terapeuta se presentan bajo la diagonal. a Esta variable se encuentra corregida a partir del logaritmo natural de la variable original. ${ }^{*} p<0,05 ;{ }^{* *} p<0,001$ 
Tabla 3

Parámetros del Modelo de Mediación de Alianza Terapéutica en la Relación entre Autocriticismo y Abandono de la Psicoterapia

\begin{tabular}{lcccrrr}
\hline \multirow{2}{*}{ Variable } & \multicolumn{3}{c}{ Alianza terapéutica ${ }^{\mathrm{a}}$} & \multicolumn{2}{c}{ Abandono psicoterapia $^{\mathrm{b}}$} \\
\cline { 2 - 7 } & \multicolumn{1}{c}{$b$} & $E E$ & $p$ & $b$ & $E E$ & $p$ \\
\hline Constante & 2,31 & 0,03 & $<0,001$ & 33,40 & 16,98 & 0,049 \\
Alianza terapéuticac (efecto b) & - & - & - & $-12,73$ & 7,18 & 0,076 \\
Autocriticismo (efecto a) & $-0,01$ & 0,01 & 0,203 & 0,56 & 0,41 & 0,179 \\
Sexo del paciente & 0,00 & 0,02 & 0,918 & $-1,13$ & 0,89 & 0,206 \\
Edad del paciente & 0,00 & 0,00 & 0,472 & $-0,11$ & 0,03 & $<0,001$ \\
Experiencia del terapeuta & 0,00 & 0,00 & 0,065 & 0,05 & 0,06 & 0,432 \\
\hline
\end{tabular}

Nota. $\mathrm{EE}=$ error estándar. ${ }^{a}$ Variable dependiente Alianza Terapéutica (a path); $R^{2}=0,08 ; F(4,76)=1,58$, $p=0,189$. ${ }^{b}$ Variable dependiente Abandono (b path) $(1=S i ́, 0=N o)$; Modelo LL $=34,94, R^{2}$ de Cox \& Snell =

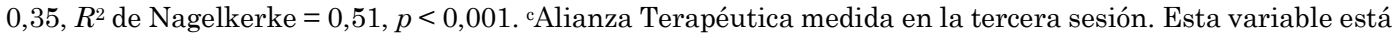
corregida a partir del logaritmo natural de la variable original. $N=81$.

\section{Relación entre Autocriticismo y Abandono Moderada por la Edad de los Pacientes}

El modelo estimado indica que, controlando el sexo y la escolaridad de los pacientes y la experiencia de los terapeutas, el nivel de autocriticismo $(b=2,33, p=0,004)$ y de dependencia $(b=-1,21, p=0,025)$ de los pacientes predicen la probabilidad de abandono de la psicoterapia. Así, a mayor autocriticismo mayor probabilidad de abandono y a mayor dependencia menor probabilidad de abandono (ver Tabla 4).

Tabla 4

Parámetros del Modelo de Moderación

\begin{tabular}{|c|c|c|c|}
\hline Variable & $b$ & $E E$ & $p$ \\
\hline Constante & $-8,02$ & $-2,96$ & 0,007 \\
\hline Edad del paciente ${ }^{a}$ & $-0,22$ & 0,08 & 0,004 \\
\hline Sexo del paciente & $-0,34$ & 1,07 & 0,754 \\
\hline Escolaridad del paciente & 0,73 & 0,36 & 0,043 \\
\hline Experiencia del terapeuta ${ }^{a}$ & 0,09 & 0,08 & 0,237 \\
\hline Autocriticismo ${ }^{\mathrm{a}}$ & 2,33 & 0,80 & 0,004 \\
\hline Dependencia $^{a}$ & $-1,21$ & 0,54 & 0,025 \\
\hline Autocriticismo $^{\mathrm{a}} \mathrm{x}$ Edad del paciente ${ }^{\mathrm{a}}$ & 0,12 & 0,06 & 0,044 \\
\hline
\end{tabular}

Nota. Variable dependiente Abandono $\left(1=S i ́, 0=N o\right.$; Modelo LL $=48,41, R^{2}$ de Cox $\&$ Snell $=0,449 ; R^{2}$ de Nagelkerke $=0,652, p<0,001, N=81$. ${ }^{\text {a }}$ Los valores para variables cuantitativas fueron centrados respecto de la media.

No obstante, la relación entre autocriticismo y la probabilidad de abandono está moderada por la edad de los pacientes. Específicamente, cuando los pacientes tienen 30 años (1 $D E$ bajo la media) su nivel de autocriticismo no predice la probabilidad de abandonar la psicoterapia $(b=0,71, p=0,280)$, en cambio, dicha relación sí se presenta cuando los pacientes tienen mayor edad. En el caso de los pacientes con 43 años de edad (edad promedio), el coeficiente de regresión es $b=2,33, p=0,004$, aumentando a $b=3,95, p=0,008$ en los pacientes de 56 años de edad (1 $D E$ sobre la media). Es decir, cuando los pacientes pertenecen a estos tramos etarios, a mayor autocriticismo mayor probabilidad de que abandonen la psicoterapia, presentándose dicho efecto más intensamente en los pacientes con edad promedio (ver Figura 1). 


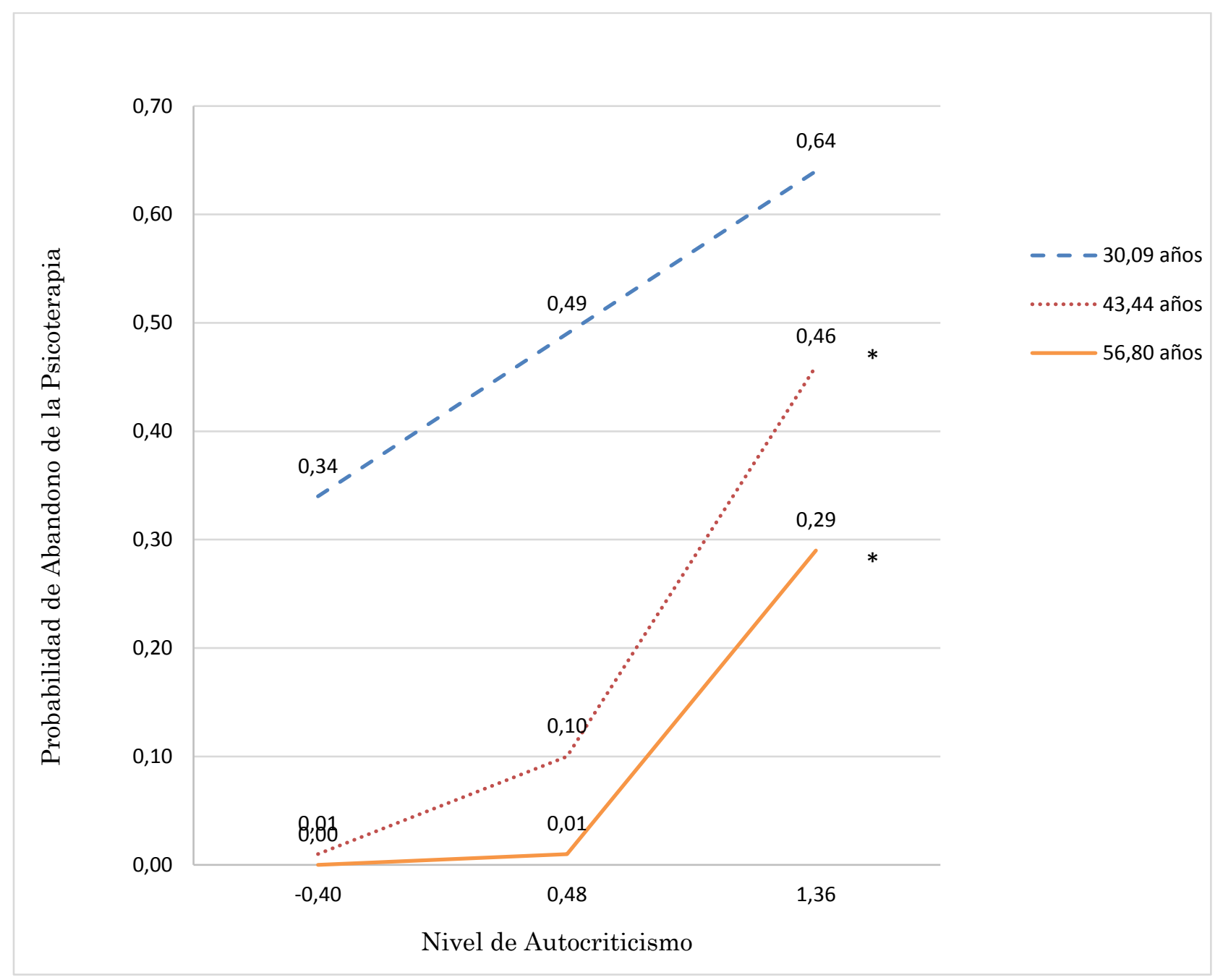

Figura 1. Probabilidad de abandono de la psicoterapia considerando el nivel de autocriticismo y la edad de los pacientes con depresión $\left({ }^{*} p<0,01\right)$.

\section{Discusión}

El objetivo central de este estudio fue describir la relación entre el autocriticismo, una distorsión relevante en el desarrollo de la personalidad de pacientes con depresión, y el abandono de psicoterapias breves destinadas a su abordaje. El porcentaje de pacientes que abandonaron la terapia (27,2\%) se acerca a las cifras internacionales de abandono (24,63\%) en pacientes con depresión (Cooper \& Conklin, 2015) y se eleva ocho puntos por sobre el porcentaje encontrado en uno de los pocos estudios que se han realizado con pacientes adultos con depresión en Chile (Alvarado et al., 2005). La comparación entre los porcentajes de ambos estudios chilenos es solo referencial, ya que fueron realizados en contextos distintos, uno con pacientes de un centro de salud privado y otro con pacientes beneficiarios de un programa del sistema público de atención primaria.

Es importante destacar que la correlación positiva encontrada en este estudio entre autocriticismo y sintomatología depresiva, la cual ya había sido reportada en otras investigaciones (Campos et al., 2010; Dagnino et al., 2017, DiBartolo et al., 2008; Dunkley et al., 2006, 2009; Enns \& Cox, 1999; Hewitt \& Flett, 1991; Rudolph et al., 2007), reafirma la necesidad de seguir integrando aspectos de la personalidad de los pacientes depresivos, más allá del plano puramente sintomatológico, en la comprensión y sistematización del abordaje psicoterapéutico de la depresión.

Asimismo, a partir de la correlación positiva encontrada en este estudio entre autocriticismo y abandono de la psicoterapia (primera hipótesis de este estudio) y la correlación inversa encontrada entre dependencia 
y abandono, se aporta con nueva evidencia respecto a que las distorsiones en la dimensión de autodefinición de la personalidad (configuración introyectiva) parecen presentarse más intensamente en las depresiones más problemáticas (Campos et al., 2010; Dunkley et al., 2006, 2009; Enns \& Cox, 1999; Hewitt \& Flett, 1991). Parece establecerse, así, una dinámica que se perpetúa en la medida en que el abandono interrumpe la posibilidad de tratamiento, afectando la gravedad del curso de la depresión. Este resultado parece sustentar, además, la necesidad de implementar procedimientos diagnósticos que puedan evaluar el nivel de autocriticismo de los pacientes antes de iniciar un tratamiento para la depresión, no solo porque los pacientes autocríticos suelen presentar resultados más pobres en las psicoterapias breves (Blatt \& Zuroff, 2005; Egan et al., 2011), sino también porque parecen existir elementos de la personalidad asociados al autocriticismo que podrían aumentar la probabilidad de abandono de la psicoterapia antes de lograr los resultados esperados. Asimismo, la relación positiva — aunque baja - encontrada entre autocriticismo y dependencia puede mostrar que la existencia de un déficit en la integración de ambas dimensiones de la personalidad puede manifestarse conjuntamente (Blatt, 2004; Blatt et al., 1976), complejizando aún más la presentación clínica de la depresión.

Un hallazgo fundamental de este estudio es el rol moderador de la edad de los pacientes entre el autocriticismo y la probabilidad de abandonar la psicoterapia, estableciendo que en los pacientes de mayor edad (1 $D E$ sobre el promedio: 56,80 años), a diferencia de aquellos menores (1 $D E$ bajo el promedio: 30,09 años), los niveles más elevados de autocriticismo se asocian con un aumento en la probabilidad de abandonar la psicoterapia. Si bien algunos estudios internacionales reportaron que uno de los predictores del abandono en pacientes adultos con depresión es pertenecer al grupo de menor edad (Arnow et al., 2007; Simon \& Ludman, 2010), considerando los resultados de este estudio, se sugiere que los pacientes de más edad, sobre todo los pacientes de edad promedio, igualmente pueden abandonar la psicoterapia, si es que presentan niveles elevados de autocriticismo y posiblemente niveles más bajos de dependencia.

Llama la atención no haber encontrado una mediación de la alianza percibida por los pacientes en la relación entre el autocriticismo y el abandono de la psicoterapia, como lo expresaba la segunda hipótesis de este estudio. Al considerar que los pacientes con altos niveles de autocriticismo tienen dificultades para establecer una buena alianza y que la alianza puede ayudar a reducir el perfeccionismo a lo largo de la terapia, convirtiéndose además en un aspecto protector frente al abandono (Egan et al., 2011; Whelton et al., 2007; Zuroff \& Blatt, 2006; Zuroff et al., 2000), fue importante estudiarlo. Ahora bien, los autores del metaanálisis que muestra una correlación inversa entre la alianza y la probabilidad de abandono de la psicoterapia (Sharf et al., 2010) sugieren que, para comprender sus resultados, es necesario observar el efecto moderador de las psicoterapias de mayor duración (16 a 40 sesiones) —intensificando dicha correlación-, señalando que los pacientes pueden verse mayormente influenciados por su relación terapéutica y abandonar por las dificultades surgidas en la alianza. De esta manera, la evaluación de la alianza al comienzo de la psicoterapia puede no ser un buen indicador para ponderar dicha correlación, ya que posiblemente no permite identificar trayectorias de la alianza asociadas al nivel de autocriticismo de los pacientes. Además, en este estudio el nivel de alianza percibida por los pacientes al inicio del tratamiento es en general muy elevado, lo que pudo disminuir su variabilidad y afectar las correlaciones evaluadas.

Retomando la línea de un eventual diagnóstico inicial en los pacientes con depresión que otorgue preponderancia a su nivel de autocriticismo, incluyendo otras dimensiones características de la personalidad, se pueden sumar algunas intervenciones clínicas iniciales que puedan utilizarse complementariamente para beneficiar su tratamiento. Por ejemplo, Shafran, Egan y Wade (2010) han elaborado un manual que sugiere un modelo cognitivo-conductual para abordar el perfeccionismo y que puede usarse diferenciadamente en los pacientes que ingresen a un tratamiento de la depresión. Egan et al. (2011) además sugieren que en pacientes con altos niveles de perfeccionismo se puede comenzar el tratamiento interviniendo directamente en la presión que estos podrían sentir por cumplir logros y que puede afectar directamente su auto-valoración, desde etapas muy tempranas de la psicoterapia. Whelton et al. (2007) proponen trabajar inicialmente temáticas de confianza, seguridad y control con estos pacientes, debido a su sensibilidad frente a la hostilidad y al particular estilo relacional que mantendrán con sus psicoterapeutas. Es decir, la sugerencia de un diagnóstico previo en este sentido puede orientar mejor los planes de acción específicos de los modelos de psicoterapia para la depresión, buscando abordar las principales problemáticas que se puedan identificar.

En este estudio el abandono se considera una finalización no deseable de la psicoterapia, aunque no necesariamente un fracaso terapéutico, ya que, como plantea Lampropoulos (2011), la definición de fracaso puede dar cuenta de distintos aspectos difíciles de agrupar en un único concepto. En este sentido, sería importante identificar con más precisión de qué se trata este tipo de abandono, ya que, tal como se presenta 
en los antecedentes, existen distintos significados al respecto. De todas maneras, aunque el abandono estuviese asociado a una instancia positiva para los pacientes o a una forma de separación de sus terapeutas una vez que se sienten bien y creen no necesitar más psicoterapia, es importante considerar que, al no dar aviso a la institución o al psicólogo tratante, esta interrupción del tratamiento puede representar un problema en la construcción del contrato y vínculo terapéuticos que puede ser necesario abordar. Los terapeutas podrían comprender (e idealmente evitar) ciertos tipos de abandono, al tener mayor claridad respecto de la distorsión autocrítica de la personalidad, lo que les puede ayudar a tratar con la incertidumbre e incluso con sentimientos de incompetencia (Piselli, Halgin \& MacEwan, 2011) derivados de esta experiencia.

Futuras investigaciones, además de precisar qué tipo de abandono puede darse en pacientes adultos con depresión, podrían identificar momentos de la terapia en la que el abandono es más preponderante. En este estudio los abandonos se produjeron en distintas etapas de tratamiento, por lo que sería importante confirmar a través de otros estudios si el nivel de autocriticismo tiene una influencia diferenciada, dependiendo del momento de la psicoterapia. La noción de abandono utilizada en este estudio se limita a un criterio institucional (alta administrativa) y del terapeuta, que no aborda las razones que tuvieron los pacientes para hacerlo, por lo que no distingue los distintos tipos de abandono que pueden encontrarse en la psicoterapia.

La interpretación de los resultados de este estudio debe tener en consideración que se trata de una muestra de pacientes que representa a aquellos que poseen un alto nivel educacional y que son beneficiarios de GES-AUGE de una institución de salud privada, por lo tanto, no representa a la gran mayoría de pacientes chilenos que padecen depresión y son atendidos en el sistema público de salud.

Por otro lado, al ser pacientes beneficiarios de GES-AUGE, estos no presentan un diagnóstico clínico de comorbilidad realizado por el psiquiatra derivante. Carecer de esta información es una limitación importante, debido al alto grado de comorbilidad entre depresión y trastorno de personalidad. Futuras investigaciones debieran incluir la evaluación de este diagnóstico u otros que podrían estar presentes y que debieran ser considerados en los análisis.

Que la participación en este estudio fuera voluntaria podría haber sesgado la muestra hacia pacientes que presentan un mayor grado de colaboración, respecto de aquellos que optan por no participar. Esta parece ser una limitación inevitable en este tipo de estudios. Esta actitud colaborativa se podría manifestar también en la psicoterapia, haciéndolos menos propensos al abandono que aquellos que eligen desde el comienzo no participar.

\section{Referencias}

Alvarado, R., Vega, J., Sanhueza, G. \& Muñoz, M. G. (2005). Evaluación del programa para la detección, diagnóstico y tratamiento integral de la depresión en atención primaria, en Chile. Revista Panamericana de Salud Pública, 18, 278-286. https://doi.org/10.1590/S102049892005000900008

Arnow, B. A., Blasey, C., Manber, R., Constantino, M. J., Markowitz, J. C., Klein, D. N. ... Rush, A. J. (2007). Dropouts versus completers among chronically depressed outpatients. Journal of Affective Disorders, 97, 197-202. https://doi.org/10.1016/j.jad.2006.06.017

Beck, A. T., Ward, C. H., Mendelson, M., Mock, J. \& Erbaugh, J. (1961). An inventory for measuring depression. Archives of General Psychiatry, 4, 561-571. https://doi.org/10.1001/archpsyc.1961.01710120031004

Blatt, S. J. (1974). Levels of object representation in anaclitic and introjective depression. The Psychoanalytic Study of the Child, 29, 107-157. https://doi.org/10.1080/00797308.1974.11822616

Blatt, S. J. (1995). The destructiveness of perfectionism: Implications for the treatment of depression. American Psychologist, 50, 1003-1020. https://doi.org/10.1037/0003-066X.50.12.1003

Blatt, S. J. (2004). Experiences of depression: Theoretical, clinical, and research perspectives. Washington, DC: American Psychological Association. https://doi.org/10.1037/10749-000

Blatt, S. J. (2007). A fundamental polarity in psychoanalysis: Implications for personality development, psychopathology, and the therapeutic process. Psychoanalytic Inquiry, 26, 494-520. https://doi.org/10.1080/07351690701310581

Blatt, S. J. (2008). Polarities of experience: Relatedness and self-definition in personality development, psychopathology, and the therapeutic process. Washington, DC: American Psychological Association. https://doi.org/10.1037/11749-000

Blatt, S. J., D'Afflitti, J. P. \& Quinlan, D. M. (1976). Experiences of depression in normal young adults. Journal of Abnormal Psychology, 85, 383-389. https://doi.org/10.1037/0021-843X.85.4.383

Blatt, S. J., D'Afflitti, J. P. \& Quinlan, D. M. (1979). Depressive Experiences Questionnaire. Unpublished manual. New Haven, CT: Yale University.

Blatt, S. J., Ford, R. Q., Berman Jr., W. H., Cook, B., Cramer, P. \& Robins, C. E. (1994). Therapeutic change: An object relations perspective. New York, NY: Plenum Press. https://doi.org/10.1007/978-1-4899-1010-3

Blatt, S. J. \& Luyten, P. (2009). Depression as an evolutionarily conserved mechanism to terminate separation distress: Only part of the biopsychosocial story? Neuropsychoanalysis, 11, 52-61. https://doi.org/10.1080/15294145.2009.10773594

Blatt, S. J., Quinlan, D. M., Chevron, E. S., McDonald, C. \& Zuroff, D. (1982). Dependency and self-criticism: Psychological dimensions of depression. Journal of Consulting and Clinical Psychology, 50, 113-124. https://doi.org/10.1037/0022-006X.50.1.113 
Blatt, S. J., Quinlan, D. M., Pilkonis, P. A. \& Shea, M. T. (1995). Impact of perfectionism and need for approval on the brief treatment of depression: The National Institute of Mental Health Treatment of Depression Collaborative Research Program revisited. Journal of Consulting and Clinical Psychology, 63, 125-132. https://doi.org/10.1037/0022-006X.63.1.125

Blatt, S. J., Shahar, G. \& Zuroff, D. C. (2001). Anaclitic (sociotropic) and introjective (autonomous) dimensions. Psychotherapy: Theory, Research, Practice, Training, 38, 449-454. https://doi.org/10.1037/0033-3204.38.4.449

Blatt, S. J. \& Zuroff, D. C. (2005). Empirical evaluation of the assumptions in identifying evidence based treatments in mental health. Clinical Psychology Review, 25, 459-486. https://doi.org/10.1016/j.cpr.2005.03.001

Blatt, S. J., Zuroff, D. C., Bondi, C. M., Sanislow III, C. A. \& Pilkonis, P. A. (1998). When and how perfectionism impedes the brief treatment of depression: Further analyses of the National Institute of Mental Health Treatment of Depression Collaborative Research Program. Journal of Consulting and Clinical Psychology, 66, 423-428. https://doi.org/10.1037/0022-006X.66.2.423

Blatt, S. J., Zuroff, D. C., Hawley, L. L. \& Auerbach, J. S. (2010). Predictors of sustained therapeutic change. Psychotherapy Research, 20, 37-54. https://doi.org/10.1080/10503300903121080

Botella, L. \& Corbella, S. (2011). Alianza terapéutica evaluada por el paciente y mejora sintomática a lo largo del proceso terapéutico. Boletín de Psicología, 101, 21-33. Extraído de http://www.uv.es/seoane/boletin/previos/N101-2.pdf

Campos, R. C., Besser, A. \& Blatt, S. J. (2010). The mediating role of self- criticism and dependency in the association between perceptions of maternal caring and depressive symptoms. Depression and Anxiety, 27, 1149-1157. https://doi.org/10.1002/da.20763

Chile, Ministerio de Salud (2001). Guía clínica para la atención primaria. La depresión: detección, diagnóstico y tratamiento. Santiago, Chile: Autor, Unidad de Salud Mental y Departamento de Modelo de Atención.

Cooper, A. A. \& Conklin, L. R. (2015). Dropout from individual psychotherapy for major depression: A meta-analysis of randomized clinical trials. Clinical Psychology Review, 40, 57-65. https://doi.org/10.1016/j.cpr.2015.05.001

Coyne, J. C. \& Whiffen, V. E. (1995). Issues in personality as diathesis for depression: The case of sociotropy-dependency and autonomyself-criticism. Psychological Bulletin, 118, 358-378. https://doi.org/10.1037/0033-2909.118.3.358

Dagnino, P., Pérez, C., Gómez, A., Gloger, S. \& Krause, M. (2017). Depression and attachment: How do personality styles and social support influence this relation? Research in Psychotherapy: Psychopathology, Process and Outcome, 20, 53-62. https://doi.org/10.4081/ripppo.2017.237

DiBartolo, P. M., Li, C. Y. \& Frost, R. O. (2008). How do the dimensions of perfectionism relate to mental health? Cognitive Therapy and Research, 32, 401-417. https://doi.org/10.1007/s10608-007-9157-7

Dunkley, D. M., Sanislow, C. A., Grilo, C. M. \& McGlashan, T. H. (2006). Perfectionism and depressive symptoms 3 years later: Negative social interactions, avoidant coping, and perceived social support as mediators. Comprehensive Psychiatry, 47, 106-115. https://doi.org/10.1016/j.comppsych.2005.06.003

Dunkley, D. M., Sanislow, C. A., Grilo, C. M. \& McGlashan, T. H. (2009). Self-criticism versus neuroticism in predicting depression and psychosocial impairment for 4 years in a clinical sample. Comprehensive Psychiatry, 50, 335-346. https://doi.org/10.1016/j.comppsych.2008.09.004

Egan, S. J., Wade, T. D. \& Shafran, R. (2011). Perfectionism as a transdiagnostic process: A clinical review. Clinical Psychology Review, 31, 203-212. https://doi.org/10.1016/j.cpr.2010.04.009

Enns, M. W. \& Cox, B. J. (1999). Perfectionism and depression symptom severity in major depressive disorder. Behaviour Research and Therapy, 37, 783-794. https://doi.org/10.1016/S0005-7967(98)00188-0

Guzmán, M. \& Mora, C. (1995). Razones de deserción desde la perspectiva del cliente y grado de mejoría y satisfacción entre los desertores tempranos versus los desertores tardíos (Tesis de Doctorado no publicada), Escuela de Psicología, Pontificia Universidad Católica de Chile, Santiago, Chile.

Hamilton, S., Moore, A. M., Crane, D. R. \& Payne, S. H. (2011). Psychotherapy dropouts: Differences by modality, license, and DSM- IV diagnosis. Journal of Marital and Family Therapy, 37, 333-343. https://doi.org/10.1111/j.1752-0606.2010.00204.x

Hans, E. \& Hiller, W. (2013). Effectiveness of and dropout from outpatient cognitive behavioral therapy for adult unipolar depression: A meta-analysis of nonrandomized effectiveness studies. Journal of Consulting and Clinical Psychology, 81, 75-88. https://doi.org/10.1037/a0031080

Hawley, L. L., Ho, M. -H. R., Zuroff, D. C. \& Blatt, S. J. (2006). The relationship of perfectionism, depression, and therapeutic alliance during treatment for depression: Latent difference score analysis. Journal of Consulting and Clinical Psychology, 74, 930-942. https://doi.org/10.1037/0022-006X.74.5.930

Hayes, A. F. (2013). Introduction to mediation, moderation, and conditional process analysis: A regression-based approach. New York, NY: Guilford Press.

Hewitt, P. L. \& Flett, G. L. (1991). Dimensions of perfectionism in unipolar depression. Journal of Abnormal Psychology, 100 , 98-101. https://doi.org/10.1037/0021-843X.100.1.98

Horvath, A. O. \& Greenberg, L. S. (1989). Development and validation of the Working Alliance Inventory. Journal of Counseling Psychology, 36, 223-233. https://doi.org/10.1037/0022-0167.36.2.223

Horvath, A. O. \& Symonds, B. D. (1991). Relation between working alliance and outcome in psychotherapy: A meta-analysis. Journal of Counseling Psychology, 38, 139-149. https://doi.org/10.1037/0022-0167.38.2.139

Krebs, M., González, L. M., Rivera, A., Herrera, P. \& Melis, F. (2012). Adherencia a psicoterapia en pacientes con trastornos de ansiedad. Psykhe, 21(2), 133-147. https://doi.org/10.7764/psykhe.21.2.550

Lampropoulos, G. K. (2011). Failure in psychotherapy: An introduction. Journal of Clinical Psychology, 67, $1093-1095$. https://doi.org/10.1002/jclp.20858

Lopes, R. T., Gonçalves, M. M., Sinai, D. \& Machado, P. P. P. (2015). Predictors of dropout in a controlled clinical trial of psychotherapy for moderate depression. International Journal of Clinical and Health Psychology, 15, 76-80. https://doi.org/10.1016/j.ijchp.2014.11.001

Luthar, S. S. \& Blatt, S. J. (1995). Differential vulnerability of dependency and self-criticism among disadvantaged teenagers. Journal of Research on Adolescence, 5, 431-449. https://doi.org/10.1207/s15327795jra0504_3

Luyten, P. \& Blatt, S. J. (2011). Psychodynamic approaches of depression: Whither shall we go? Psychiatry: Interpersonal and Biological Processes, 74, 1-3. https://doi.org/10.1521/psyc.2011.74.1.1

Luyten, P. \& Blatt, S. J. (2015). The psychodynamic approach to diagnosis and classification. En P. Luyten, L. C. Mayes, P. Fonagy, M. Target \& S. J. Blatt (Eds.), Handbook of psychodynamic approaches to psychopathology psychopathology (pp. 87-109). New York, NY: Guilford Press. 
Luyten, P., Corveleyn, J. \& Blatt, S. J. (2005). The convergence among psychodynamic and cognitive-behavioral theories of depression: A critical review of empirical research. En J. Corveleyn, P. Luyten \& S. J. Blatt (Eds.), The theory and treatment of depression: Towards a dynamic interaccionism model (pp. 95-135). Leuven, Bélgica: Leuven University Press \& Mahwah, NJ: Lawrence Erlbaum.

Luyten, P., Sabbe, B., Blatt, S. J., Meganck, S., Jansen, B., De Grave, C. ... Corveleyn, J. (2007). Dependency and self- criticism: Relationship with major depressive disorder, severity of depression, and clinical presentation. Depression and Anxiety, 24, 586-596. https://doi.org/10.1002/da.20272

Menéndez, P. Á., Acosta, S. R., Bodon, M. C. \& Mayorga, P. M. (2010). Descripción de observables clínicos y de cambios en el proceso de admisión y de su impacto en el inicio del tratamiento psicoterapéutico. Anuario de Investigaciones, 17, 49-57. Extraído de http://www.aacademica.org/000-032/154.pdf

Ogrodniczuk, J. S., Joyce, A. S. \& Piper, W. E. (2005). Strategies for reducing patient-initiated premature termination of psychotherapy. Harvard Review of Psychiatry, 13, 57-70. https://doi.org/10.1080/10673220590956429

Persons, J. B., Burns, D. D. \& Perloff, J. M. (1988). Predictors of dropout and outcome in cognitive therapy for depression in a private practice setting. Cognitive Therapy and Research, 12, 557-575. https://doi.org/10.1007/BF01205010

Piselli, A., Halgin, R. P. \& MacEwan, G. H. (2011). What went wrong? Therapists' reflections on their role in premature termination. Psychotherapy Research, 21, 400-415. https://doi.org/10.1080/10503307.2011.573819

Porcel Medina, M. (2005). El abandono en las terapias psicológicas. Aposta: Revista de Ciencias Sociales, 14, 1-16. Extraído de http://www.apostadigital.com/revistav3/hemeroteca/porcel.pdf

Preacher, K. J. \& Hayes, A. F. (2008). Asymptotic and resampling strategies for assessing and comparing indirect effects in multiple mediator models. Behavior Research Methods, 40, 879-891. https://doi.org/10.3758/BRM.40.3.879

Rost, R. \& Dagnino, P. (2011). Traducción a Chile del Depressive Experience Questionnaire (DEQ). Manuscrito no publicado, Pontificia Universidad Catolica de Chile, Santiago, Chile.

Rudolph, S. G., Flett, G. L. \& Hewitt, P. L. (2007). Perfectionism and deficits in cognitive emotion regulation. Journal of RationalEmotive \& Cognitive-Behavior Therapy, 25, 343-357. https://doi.org/10.1007/s10942-007-0056-3

Santibáñez, P. (2003). La alianza terapéutica en psicoterapia: el "Inventario de Alianza de Trabajo" en Chile. Psykhe, 12(1), 109-118. Extraído de http://www.psykhe.cl/index.php/psykhe/article/view/344/324

Schindler, A., Hiller, W. \& Witthöft, M. (2013). What predicts outcome, response, and drop-out in CBT of depressive adults? A naturalistic study. Behavioural and Cognitive Psychotherapy, 41, 365-370. https://doi.org/10.1017/S1352465812001063

Shafran, R., Egan, S. \& Wade, T. (2010). Overcoming perfectionism: A self-help guide using cognitive behavioral techniques. London, Reino Unido: Constable \& Robinson.

Shahar, G. (2006). An investigation of the perfectionism/self-criticism domain of the Personal Style Inventory. Cognitive Therapy and Research, 30, 185-200. https://doi.org/10.1007/s10608-006-9032-y

Shahar, G., Blatt, S. J. \& Zuroff, D. C. (2007). Satisfaction with social relations buffers the adverse effect of (mid-level) selfcritical perfectionism in brief treatment for depression. Journal of Social \& Clinical Psychology, 26, 540-555. https://doi.org/10.1521/jscp.2007.26.5.540

Shahar, G., Blatt, S. J., Zuroff, D. C., Krupnick, J. L. \& Sotsky, S. M. (2004). Perfectionism impedes social relations and response to brief treatment for depression. Journal of Social \& Clinical Psychology, 23, 140-154. https://doi.org/10.1521/jscp.23.2.140.31017

Shahar, G., Joiner Jr., T. E., Zuroff, D. C. \& Blatt, S. J. (2004). Personality, interpersonal behavior, and depression: Co existence of stress-specific moderating and mediating effects. Personality and Individual Differences, 36, $1583-1596$. https://doi.org/10.1016/j.paid.2003.06.006

Sharf, J. (2008). Psychotherapy dropout: A meta-analytic review of premature termination (Tesis de Doctorado). Extraído de la base de Dissertation Abstracts International Section B: The Sciences and Engineering, 68(9-B), 6336.

Sharf, J., Primavera, L. H. \& Diener, M. J. (2010). Dropout and therapeutic alliance: A meta-analysis of adult individual psychotherapy. Psychotherapy: Theory, Research, Practice, Training, 47, 637-645. https://doi.org/10.1037/a0021175

Simon, G. E. \& Ludman, E. J. (2010). Predictors of early dropout from psychotherapy for depression in community practice. Psychiatric Services, 61, 684-689. https://doi.org/10.1176/ps.2010.61.7.684

Swift, J. K. \& Greenberg, R. P. (2012). Premature discontinuation in adult psychotherapy: A meta-analysis. Journal of Consulting and Clinical Psychology, 80, 547-559. https://doi.org/10.1037/a0028226

Swift, J. K. \& Greenberg, R. P. (2014). A treatment by disorder meta-analysis of dropout from psychotherapy. Journal of Psychotherapy Integration, 24, 193-207. https://doi.org/10.1037/a0037512

Tryon, G. S. \& Kane, A. S. (1995). Client involvement, working alliance, and type of therapy termination. Psychotherapy Research, 5, 189-198. https://doi.org/10.1080/10503309512331331306

Valdés, C., Morales-Reyes, I., Pérez, J. C., Medellín, A., Rojas, G. \& Krause, M. (2017). Propiedades psicométricas del Inventario de Depresión de Beck IA para la población chilena. Revista Médica de Chile, 145, 1005-1012. https://doi.org/10.4067/s003498872017000801005

Watson, J. C. (2011). Treatment failure in humanistic and experiential psychotherapy. Journal of Clinical Psychology, 67, 1117-1128. https://doi.org/10.1002/jclp.20849

Whelton, W. J., Paulson, B. \& Marusiak, C. W. (2007). Self-criticism and the therapeutic relationship. Counselling Psychology Quarterly, 20, 135-148. https://doi.org/10.1080/09515070701412423

Wierzbicki, M. \& Pekarik, G. (1993). A meta-analysis of psychotherapy dropout. Professional Psychology: Research and Practice, 24, 190-195. https://doi.org/10.1037/0735-7028.24.2.190

Zuroff, D. C. \& Blatt, S. J. (2006). The therapeutic relationship in the brief treatment of depression: Contributions to clinical improvement and enhanced adaptive capacities. Journal of Consulting and Clinical Psychology, 74, 130-140. https://doi.org/10.1037/0022006X.74.1.130

Zuroff, D. C., Blatt, S. J., Sotsky, S. M., Krupnick, J. L., Martin, D. J., Sanislow III, C. A. \& Simmens, S. (2000). Relation of therapeutic alliance and perfectionism to outcome in brief outpatient treatment of depression. Journal of Consulting and Clinical Psychology, 68, 114-124. https://doi.org/10.1037/0022-006X.68.1.114

Zuroff, D. C. \& Mongrain, M. (1987). Dependency and self-criticism: Vulnerability factors for depressive affective states. Journal of Abnormal Psychology, 96, 14-22. https://doi.org/10.1037/0021-843X.96.1.14 
Zuroff, D. C., Moskowitz, D. S., Wielgus, M. S., Powers, T. A. \& Franko, D. L. (1983). Construct validation of the dependency and self-criticism scales of the Depressive Experiences Questionnaire. Journal of Research in Personality, 17, 226-241. https://doi.org/10.1016/00926566(83)90033-8

Zuroff, D. C., Quinlan, D. M. \& Blatt, S. J. (1990). Psychometric properties of the Depressive Experiences Questionnaire in a college population. Journal of Personality Assessment, 55, 65-72. https://doi.org/10.1080/00223891.1990.9674047

Fecha de recepción: Diciembre de 2016.

Fecha de aceptación: Octubre de 2018. 